\title{
Erratum: Factors influencing patient falls in a private hospital group in the Cape Metropole of the Western Cape
}

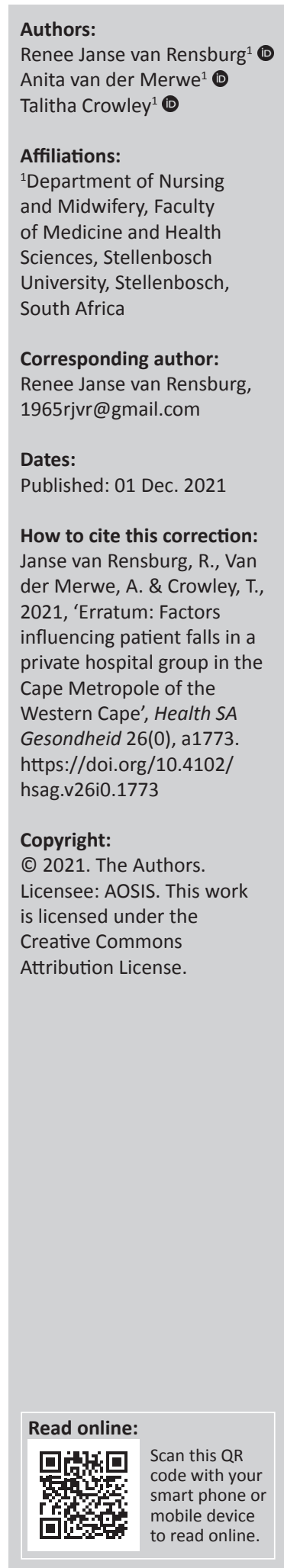

\section{Authors:}

Anita van der Merwe ${ }^{1}$ (D)

Talitha Crowley ${ }^{1}$ (D)

\section{Affiliations:}

Department of Nursing

of Medicine and Health

Sciences, Stellenbosch

University, Stellenbosch,

South Africa

Corresponding author:

Renee Janse van Rensburg,

1965rjvr@gmail.com

How to cite this correction:

2021, 'Erratum: Factors

influencing patient falls in a

Cape Metropole of the

Western Cape', Health SA

Gesondheid 26(0), a1773.

https://doi.org/10.4102/
In the version of this article initially published, Janse van Rensburg, R., Van der Merwe, A. \& Crowley, T., 2020, 'Factors influencing patient falls in a private hospital group in the Cape Metropole of the Western Cape', Health SA Gesondheid 25(0), a1392. https://doi.org/10.4102/ hsag.v25i0.1392, the article section was given incorrectly. The correct section should be Original Research instead of Review Article.

This correction does not alter the study's findings of significance or overall interpretation of the study's results. The publisher apologises for any inconvenience caused. 